\title{
INVESTIGACIONES
}

\section{Diseño y validación de un Escala de Inmersión Digital para Adolescentes*}

\author{
Development and validation of a Digital Immersion Scale \\ for Adolescents
}

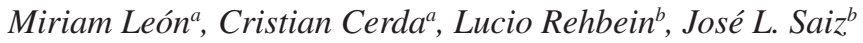 \\ ${ }^{a}$ Departamento de Educación, Universidad de La Frontera, Temuco, Chile. \\ miriam.leon@ufrontera.cl, cristian.cerda@ufrontera.cl \\ ${ }^{b}$ Departamento de Psicología, Universidad de La Frontera, Temuco, Chile \\ lucio.rehbein@ufrontera.cl,jose.saiz@ufrontera.cl
}

\section{RESUMEN}

El objetivo de este estudio fue construir y analizar psicométricamente una escala para medir inmersión digital en adolescentes. Se definió la inmersión digital como el uso persistente, continuo y entusiasta de dispositivos y medios digitales acompañado de la capacidad de realizar múltiples tareas de manera simultánea, con sentimientos de autoeficacia, confianza, dependencia e identificación con la tecnología. Se analizó la validez de contenido a través de juicio de expertos ( $\mathrm{n}=8$ ); luego se realizaron análisis de validez de la estructura interna mediante Análisis Factorial Exploratorio ( $n=347)$ y Análisis Factorial Confirmatorio $(n=347)$. La versión definitiva de la Escala de Inmersión Digital para Adolescentes (EIDA) quedó constituida por 30 ítems, agrupados en cinco dimensiones: Variedad de Uso; Intensidad de Uso; Multitarea; Involucramiento; y Dependencia. La consistencia interna de la escala fue alta $(\alpha=.951)$ y sus resultados explican el $89 \%$ de la varianza.

Palabras clave: tecnología educacional, informática educativa, adolescencia, enseñanza secundaria, estadística.

\section{ABSTRACT}

The objective of this study was to build and psychometrically analyze a scale to measure digital immersion in adolescents. Digital immersion was defined as the persistent, continuous and enthusiastic use of devices and digital media accompanied by the ability to perform multiple tasks simultaneously with feelings self-efficacy, reliance, dependence, and identification with technology. Content validity was analyzed through expert judgment $(n=8)$; then, evidence of the validity of the internal structure was obtained through Exploratory Factor Analysis $(n=347)$ and Confirmatory Factor Analysis $(n=347)$. The final version of the Scale of Digital Immersion for Adolescents (EIDA) contained 30 items, grouped into five dimensions: Variety of Use; Intensity of Use; Multitasking: Involvement; and Dependence, explaining $89 \%$ of the variance presented in the data. The internal consistency of the scale was satisfactory $(\alpha=.951)$.

Key words: educational technology, computer uses in education, adolescence, secondary education, Statistics.

\footnotetext{
* Este estudio fue financiado por el Fondo Nacional de Desarrollo Científico y Tecnológico de Chile, Proyecto FONDECYT $\mathrm{N}^{\circ} 1191193$
} 


\section{INTRODUCCIÓN}

En los últimos años, las tecnologías digitales han pasado a ser parte constitutiva de la vida cotidiana de los jóvenes. Es muy difícil para los jóvenes actuales imaginar su vida sin mensajería instantánea y sin navegación por Internet. La disponibilidad de una amplia variedad de dispositivos multimediales está moldeando la manera en que la sociedad contemporánea genera significados, se comunica y expresa su creatividad (Ito et al., 2009). Los adolescentes de hoy están creciendo en un entorno en el que muchos aspectos de sus vidas están mediados por las tecnologías digitales (Boyd, 2014). Las tecnologías digitales entonces están cambiando la forma como nos relacionamos en sociedad.

Las tecnologías digitales están cambiando la cultura actual. Estas herramientas abarcan tanto los artefactos y los sistemas de significación y comunicación que demarcan más claramente el modo de vida contemporáneo (Gere, 2008). En otras palabras, se está frente a lo que se debería denominar, en el sentido más amplio del término, una nueva cultura: la cultura digital (Bruni, 2011). Hoy en día ser usuario de tecnologías digitales es estar inmerso en ellas.

El uso del concepto inmersión, ligado a las tecnologías digitales, está todavía en desarrollo. Una de las primeras autoras que comenzó a utilizar este concepto asociado a las tecnologías fue Murray (1997), quien señaló que la inmersión es un término metafórico derivado de la experiencia física de estar sumergido en el agua, que implica aprender a hacer las cosas que el nuevo ambiente hace posible. Este concepto se ha asociado mayoritariamente al uso de videojuegos, los mundos virtuales basados en avatar y la realidad virtual. Sin embargo, se observa una falta de consenso respecto a su definición, así como la falta de instrumentos para medirlo (Georgiou y Kyza, 2017).

Según Nilsson et al. (2016), las actuales definiciones de inmersión se pueden clasificar en cuatro tipos: (i) propiedad del sistema; (ii) respuesta perceptiva a ese sistema; (iii) respuesta subjetiva a los contenidos narrativos; y (iv) respuesta subjetiva a los desafíos cognitivos que representan las tecnologías. En propiedad del sistema, Slater (2003) señala que la inmersión es una propiedad de la tecnología, y que representa lo que esta ofrece desde un punto de vista objetivo. Esto implica que puede ser medible dado que una tecnología permite una mayor inmersión cuánto más ofrece. Por ejemplo, si la interfaz con la que se interactúa posee un buen nivel de resolución, mayor será su capacidad para inducir inmersión. En la segunda perspectiva, inmersión como respuesta perceptiva a ese sistema, Dominic (2005), lo asocia a la sensación de estar envuelto por la representación multisensorial del mundo virtual entregado a través de pantallas de alta fidelidad. En el tercer tipo de inmersión, respuesta subjetiva a los contenidos narrativos, Ermi y Mäyrä (2005) la conceptualizan como la sensación de estar mentalmente absorto. Finalmente, en inmersión como respuesta subjetiva a los desafíos cognitivos que representan las tecnologías, Ryan (2008) lo define como un estado intenso de absorción en la tarea que se realiza. Además, Adams y Rollings (2006) lo describen como un estado de intensa preocupación con respuestas rápidas a los obstáculos en los cuales se desarrollan acciones como observación, cálculo y planificación en el uso de videojuegos. Si bien se asocia inmersión a los videos juegos, existen otros autores que explícitamente mencionan el concepto de inmersión digital.

Se ha utilizado el concepto de inmersión digital asociándolo al uso persistente, continuo y entusiasta de dispositivos y medios digitales. Rosenfeld (2015) lo define como 
la amplitud y profundidad del uso de dispositivos digitales en la vida de las personas y el tiempo que pasan en el ciberespacio. Owston (2009) sugiere que la inmersión digital se refiere al tiempo que las personas invierten en actividades Web 2.0 combinado con el uso de otros medios digitales. Jenkins (2006), asoció la inmersión digital con el uso generalizado, frecuente e intenso de las tecnologías digitales. Si bien estas definiciones se concentran exclusivamente en el uso de tecnologías digitales, el concepto de inmersión digital empieza a considerar también otros aspectos más internos del sujeto.

Entre dichos aspectos destaca la incorporación de componentes como el involucramiento cognitivo y la identidad tecnológica. El involucramiento cognitivo se refleja en la focalización de la atención (Georgiou y Kyza, 2017) y en el desarrollo de habilidades multitarea (Courage et al., 2015). Por otra parte, en la identidad tecnológica, se considera la forma en que los adolescentes adoptan el uso de las tecnologías como una parte integral de sí mismos, con sentimientos de autoeficacia, confianza, dependencia e identificación con la tecnología (Carter y Grover, 2015). Los mismos autores establecen que la identidad tecnológica se refleja en tres dimensiones interrelacionadas: Compenetración, Compromiso Emocional y Dependencia. La Compenetración representa el desdibujamiento de los límites entre el sí mismo y las tecnologías, y se manifiesta como sentimientos de unión con ellas. Las personas que sienten una conexión fuerte con una tecnología la llevan consigo en todo momento y la usan en una variedad de situaciones cotidianas. El Compromiso Emocional representa los sentimientos de confianza y entusiasmo de las personas cuando piensan en sí mismos en relación con las tecnologías. En tanto, la Dependencia se refiere a como las personas confían, dependen y organizan su vida cotidiana utilizando las tecnologías. Como se ha podido observar existen distintos autores que definen inmersión considerando distintos aspectos.

El contraste entre las definiciones existentes de inmersión digital, y las evidencias de uso de las tecnologías por las nuevas generaciones, pone de manifiesto que las definiciones actuales son estrechas y parciales y requieren ser actualizadas. En este contexto, y para esta investigación, se definió inmersión digital como la amplitud, variedad e intensidad de uso (uso de tecnologías digitales); focalización preferencial y multitarea (involucramiento cognitivo), junto con la compenetración, compromiso emocional y la dependencia (identidad tecnológica). Se considera este constructo como una conducta que evidencia la forma en que las personas se desenvuelven en un ambiente rodeado de dispositivos digitales.

A partir de los antecedentes anteriormente revisados, se procedió a identificar las dimensiones y subdimensiones que se presentan en la Tabla 1. 
Tabla 1. Dimensiones teóricas para la Escala de Inmersión Digital para Adolescentes (EIDA)

\begin{tabular}{|l|l|l|}
\hline Dimensión & Sub dimensión & \multicolumn{1}{|c|}{ Breve Definición } \\
\hline \multirow{4}{*}{$\begin{array}{l}\text { Uso de } \\
\text { Tecnologías } \\
\text { Digitales }\end{array}$} & Amplitud de Uso & $\begin{array}{l}\text { Uso de distintos dispositivos tecnológicos (computador, } \\
\text { celular, Tablet, etc.). }\end{array}$ \\
\cline { 2 - 3 } & Variedad de Uso & $\begin{array}{l}\text { Uso variado de las tecnologías en distintos tipos de } \\
\text { actividades. }\end{array}$ \\
\cline { 2 - 3 } $\begin{array}{l}\text { Involucramiento } \\
\text { cognitivo }\end{array}$ & $\begin{array}{l}\text { Focalización } \\
\text { Preferencial }\end{array}$ & $\begin{array}{l}\text { Focalización preferencial en actividades realizadas con } \\
\text { tecnologías. }\end{array}$ \\
\cline { 2 - 3 } & Multitarea & Realización de varias actividades al mismo tiempo. \\
\hline \multirow{3}{*}{$\begin{array}{l}\text { Identidad } \\
\text { tecnológica }\end{array}$} & $\begin{array}{l}\text { Compenetración } \\
\text { Empromiso }\end{array}$ & $\begin{array}{l}\text { Disolución de límites entre el sí mismo y las tecnologías. } \\
\text { Se manifiesta como sentimientos de unión con ellas. }\end{array}$ \\
\cline { 2 - 3 } & Emocional & $\begin{array}{l}\text { Expresión de sentimientos de confianza, entusiasmo y } \\
\text { energía cuando una persona piensa en sí misma en relación } \\
\text { a la tecnología. }\end{array}$ \\
\cline { 2 - 3 } & Dependencia & $\begin{array}{l}\text { Expresión de sentimientos de dependencia con respecto a } \\
\text { las tecnologías. }\end{array}$ \\
\hline
\end{tabular}

Fuente. Elaboración propia.

Considerando la necesidad de conocer si esta construcción teórica de la inmersión digital tenía un correlato empírico, el objetivo del presente estudio consistió en construir y analizar psicométricamente una escala para medir inmersión digital en adolescentes. Específicamente, se planteó: determinar evidencias de validez de contenido del instrumento; determinar evidencias de validez de la estructura interna de la escala; e identificar la consistencia interna de la Escala de Inmersión Digital para Adolescentes (EIDA).

Contar con un instrumento que mida inmersión digital en adolescentes representa múltiples ventajas. Primero, permitiría comparar el nivel de inmersión digital de jóvenes de distintos contextos culturales y sociales. Por otra parte, dado que los adolescentes de las generaciones recientes son genuinamente "nativos digitales" (ya que no conocieron una época sin acceso a Internet y a las tecnologías digitales), son los mejores exponentes para dimensionar la amplitud y profundidad de la inmersión digital. El uso de tecnologías digitales en adolescentes se da en un contexto de desarrollo personal que se caracteriza por cambios sociales, cognitivos, biológicos y psicológicos en la vida de las personas (Giedd, 2012). Es relevante entonces estudiar como la inmersión digital puede ser medida en dicho contexto. 


\section{MÉTODO}

\subsection{PARTICIPANTES}

La muestra de este estudio estuvo compuesta por 694 adolescentes de la región de La Araucanía, Chile. Los participantes fueron seleccionados mediante muestreo no probabilístico por conveniencia. Como criterio de inclusión se consideró que pertenecieran a establecimientos educacionales con distintos niveles socioeconómicos y que manifestaran interés por participar en el estudio. Se contó con el consentimiento de los padres de los participantes. Por otra parte, se excluyó a adolescentes que presentaban necesidades educativas especiales asociadas a discapacidad, o a condiciones consideradas inhabilitantes por los profesores y/o los propios padres. Para efectos del análisis, esta muestra fue dividida aleatoriamente en dos mitades: los datos aportados por los primeros 347 participantes fueron sometidos a Análisis Factorial Exploratorio (AFE); y los datos de los restantes 347 participantes fueron parte del Análisis Factorial Confirmatorio (AFC). Tal como se observa en la Tabla 2, en ambas submuestras se contó con participantes con características equivalentes.

Tabla 2. Perfil demográfico muestra adolescentes $\mathrm{N}=694$

\begin{tabular}{|l|c|c|c|c|}
\hline \multirow{2}{*}{ Característica } & \multicolumn{3}{|c|}{ AFE n=347 } & \multicolumn{2}{c|}{ AFC n=347 } \\
\cline { 2 - 5 } & $\mathrm{n}$ & $\%$ & $\mathrm{n}$ & $\%$ \\
\hline Sexo & 174 & 50.1 & 175 & 50.4 \\
\hline Hombre & 173 & 49.9 & 172 & 49.6 \\
\hline Mujer & 90 & 25.9 & 98 & 28.3 \\
\hline Curso (secundaria) & 95 & 27.5 & 97 & 28.0 \\
\hline I medio & 75 & 21.8 & 74 & 21.4 \\
\hline II medio & 87 & 25.1 & 78 & 22.5 \\
\hline III medio & 92 & 26.5 & 90 & 25.9 \\
\hline IV medio & 252 & 72.6 & 254 & 73.2 \\
\hline Etnia & 3 & 0.9 & 3 & 0.9 \\
\hline Mapuche & 58 & 16.7 & 70 & 20.2 \\
\hline No mapuche & 50 & 14.4 & 64 & 18.4 \\
\hline Otra etnia & 70 & 20.2 & 53 & 15.3 \\
\hline Nivel socioeconómico & 169 & 48.7 & 160 & 46.1 \\
\hline Alto & & \\
\hline Medio & & & \\
\hline Medio bajo & \multicolumn{5}{|l|}{} \\
\hline Bajo & &
\end{tabular}

Fuente. Elaboración propia. 


\subsection{INSTRUMENTOS}

La construcción de la versión definitiva de la Escala de Inmersión Digital para Adolescentes (EIDA) requirió la revisión de varias versiones previas. Originalmente se partió con una versión de 69 ítems, que fue ampliada a una con 73 afirmaciones, luego de la revisión del juicio de expertos. Del análisis de dichos ítems, se definió una escala final con 30 aseveraciones, las que fueron seleccionadas considerando diferentes criterios. A continuación, se describe brevemente la evolución de la escala inicial de 69 ítems a su versión definitiva de 30.

\section{Versión preliminar de la Escala de Inmersión Digital para Adolescentes}

La primera versión del instrumento constó de 69 ítems. Ocho ítems pertenecientes a la dimensión Amplitud; nueve a Intensidad de Uso; nueve a Variedad de Uso; diez a Focalización; diez a Multitarea; nueve a Compenetración; nueve a Compromiso Emocional; y nueve a Dependencia. Estos ítems corresponden a afirmaciones descriptivas de conductas que se consideran son un buen reflejo de inmersión digital. La mayor parte de los ítems fueron elaborados por los autores, a partir del análisis de antecedentes empíricos descritos en la literatura y de entrevistas realizadas a estudiantes identificados por sus compañeros como líderes en el uso de las tecnologías. Otros ítems fueron tomados de escalas relacionadas al constructo (Georgiou y Kyza, 2017; Poposki y Oswald, 2010; van Deursen et al., 2014). Estos ítems fueron traducidos y retraducidos por dos expertos bilingües, y los contenidos fueron descentrados para lograr que reflejaran las conductas, tal como se manifiestan en la realidad cultural de la población juvenil chilena. Todos los reactivos presentaban un continuo de cinco opciones tipo Likert: "Muy en desacuerdo"; "En desacuerdo"; "Ni de acuerdo ni en desacuerdo"; "De acuerdo"; "Muy de acuerdo". Los ítems utilizados fueron sometidos a un proceso de evaluación.

\section{Evaluación de ítems por jueces}

Los ítems utilizados en la escala preliminar fueron sometidos a evaluación por juicio de expertos. Para el registro del juicio de los expertos se utilizó una pauta donde se presentaron los 69 ítems de la versión preliminar de la EIDA. La pauta contenía nombres y definiciones conceptuales de las dimensiones y subdimensiones de inmersión, seguidas por el conjunto de los ítems. Los jueces tenían que señalar qué tan adecuado consideraban cada ítem, en respuesta tipo Likert, que variaba de 1(Inadecuado) a 5 (Muy adecuado). Además, se entregó la opción de realizar comentarios narrativos sobre los ítems.

\section{Versión Experimental Escala de Inmersión Digital para Adolescentes}

El análisis desarrollado en la evaluación de jueces permitió tomar algunas decisiones sobre los ítems. En consideración a las recomendaciones del juicio de expertos se eliminaron 8 ítems de la versión preliminar, dado que no se considerados adecuados. Adicionalmente, se agregaron 12 ítems sugeridos por los jueces, para complementar la escala. Además, se revisó la redacción de los ítems que tuvieron porcentajes menores en cuanto a su valoración. En consecuencia, la versión experimental de la escala, con la cual se realizaron los análisis factoriales, quedó compuesta por 73 ítems. 


\section{Versión Definitiva Escala de Inmersión Digital para Adolescentes (EIDA).}

La versión definitiva de la escala está conformada por 30 ítems organizados en 5 subdimensiones: Variedad de Uso, Intensidad de Uso, Multitarea, Involucramiento y Dependencia Tecnológica. Esta estructura fue generada considerando como criterios la correspondencia teórica de cada ítem a los constructos a los cuales pertenecen, junto con las recomendaciones obtenidas a través de los análisis estadísticos realizados. Dichos análisis consideraron que la definición de una escala más breve representa una manera más eficaz y precisa de medir el constructo inmersión digital.

\subsection{PROCEDIMIENTO}

Para el proceso de evaluación de jueces expertos, se obtuvo la contribución de ocho profesionales vinculados al área de la psicología y/o educación, quienes contaban con al menos grado de magíster y conocían de la temática de uso de tecnologías digitales en educación. A los expertos se les envió vía plataforma en línea la Pauta de Evaluación, para que pudiesen realizar sus comentarios y/o sugerencias.

Para acceder a la muestra de adolescentes, se solicitó la colaboración a directivos de establecimientos educacionales de la región de La Araucanía, Chile. Ellos facilitaron la asistencia a reuniones de padres y apoderados para presentar los antecedentes del estudio, señalar el mantenimiento de confidencialidad sobre la información manejada, privacidad y voluntariedad del estudio.

Previo a la aplicación del instrumento, padres y/o apoderados firmaron un consentimiento informado, aprobado por el Comité Ético Científico de la Universidad de La Frontera. Una vez que los padres y/o apoderados firmaron el consentimiento informado, se entregó a los adolescentes el asentimiento informado, documento que les permitió conocer la investigación e informarse sobre la confidencialidad, privacidad y voluntariedad de participar en el estudio. La aplicación se realizó en dependencias de cada uno de los establecimientos educacionales participantes, previo acuerdo con los docentes de las asignaturas. Los datos obtenidos fueron digitalizados en una hoja de cálculo y, previo a su exportación a los programas estadísticos utilizados, se efectuaron las acciones de depuración y control de calidad de los datos, conforme a lo recomendado por Muñiz y Fonseca-Pedrero (2008).

\subsection{ANÁLISIS DE DATOS}

La siguiente sección presenta una descripción detallada del análisis de datos realizados. En el caso del juicio de expertos, se examinó estadísticamente el grado de acuerdo inter-jueces utilizado en la evaluación de las definiciones de los constructos y del contenido de los ítems. Para dicha tarea se calcularon los coeficientes de correlación intraclase (Rc) utilizando la fórmula ICC (A, k) para modelos Caso 2 de McGraw y Wong (1996). Posterior a estos análisis, se realizaron los análisis de validez de constructo a través de técnicas exploratorias y confirmatorias, dividiendo la muestra de participantes en dos mitades. Una muestra de 347 estudiantes para el Análisis Factorial Exploratorio (AFE) y otra también con 347 participantes para el Análisis Factorial Confirmatorio (AFC). La división de la muestra se realizó al azar, considerando que se mantuviese de manera proporcional la cantidad de hombres y mujeres en cada submuestra. 
El Análisis Factorial Exploratorio se realizó para explorar la estructura preliminar de la escala. Este análisis fue desarrollado con el programa FACTOR (Lorenzo-Seva y Ferrando, 2006), utilizando procedimientos como la versión de implementación óptima del análisis paralelo, para identificar el número de factores a extraer (Timmerman y Lorenzo-Seva, 2011); la extracción de factores mediante análisis factorial de rango mínimo (MRFA en su sigla en inglés) (Shapiro y Berge, 2002), y el uso de una rotación oblicua Promin (LorenzoSeva, 1999). En los casos en que el método de extracción (MRFA) no convergió, se utilizó el método de mínimos cuadrados no ponderados (ULS en su sigla en inglés). Atendiendo la ordinalidad de los datos, la matriz de correlaciones analizada fue la matriz policórica para valores ordinales, como lo recomiendan Flora y Curran (2004), a diferencia de otros análisis factoriales exploratorios que analizan la matriz de correlación de Pearson. El ajuste del modelo factorial emergente fue inspeccionado a través del índice de la raíz cuadrada, mediante la observación de los residuales (RMSR en su sigla en inglés). El criterio utilizado para la retención de ítems consideró cargas factoriales iguales o superiores a .30 .

El Análisis Factorial Confirmatorio (AFC) fue desarrollando utilizando el programa estadístico Stata 15. El uso de este programa permitió que la estimación de los parámetros del modelo fuera realizada usando el método de estimación de máxima verosimilitud (Maximum Likelihood). Además, debido a que el supuesto de normalidad multivariada (Análisis de Mardia) no fue obtenido, la estimación fue realizada con la corrección de Satorra-Bentler, disponible en este programa. Para la evaluación del modelo, se usaron los siguientes índices de bondad de ajuste: Media Estandarizada de la Raíz Cuadrática Residual (SRMR < .08); Índice de Tucker Lewis (TLI > .90), Índice de Ajuste Comparativo (CFI > .90); y Error Cuadrático Medio de Aproximación (RMSEA $\leq .06$ ) (Abad et al., 2011). La consistencia interna fue examinada mediante el coeficiente alfa ordinal (Zumbo et al., 2007), calculado de acuerdo al procedimiento indicado por Domínguez (2012) para escalas con variables ordinales.

\section{RESULTADOS}

A continuación, se presentan los resultados obtenidos a través de los análisis de validez de contenido de los ítems (juicio de experto), validez de la estructura interna (Análisis Factorial Exploratorio y Confirmatorio) y análisis de confiabilidad mediante consistencia interna.

\subsection{EVIDENCIAS DE VALIDEZ DE CONTENIDO DE LA ESCALA}

El análisis inter-jueces, con respecto a los ítems contenidos en la versión preliminar de la escala tuvo un amplio consenso. La evaluación de las definiciones de los sub dimensiones y el contenido de los ítems presentó los siguientes coeficientes de correlación intraclase: Sub dimensión Amplitud presentó $\mathrm{Rc}=.807, p=.001 ;$ Intensidad de Uso $(\mathrm{Rc}=.802, p=.002)$; Variedad de Uso $(\mathrm{Rc}=.890, p=.002) ;$ Focalización $(\mathrm{Rc}=.779, p<.001) ;$ Multitarea $(\mathrm{Rc}=$ $.76, p<.001)$; Compenetración $(\mathrm{Rc}=.916, p<.001)$; Compromiso Emocional $(\mathrm{Rc}=.732$, $p=.001)$; y Dependencia $\mathrm{Rc}=.763, p=.003)$. De acuerdo a los criterios de Rosner (2011), aquellos valores iguales o superiores a .75 indican un excelente grado de consenso entre los jueces. En tanto, valores entre .40 y .75 indican acuerdos regulares a buenos, mientras que valores menores a .40 indican malos consensos. Por lo tanto, los resultados obtenidos 
muestran que en la mayoría de las dimensiones hubo excelentes grados de consenso entre los jueces.

\subsection{EVIDENCIAS DE VALIDEZ DE LA ESTRUCTURA INTERNA}

\subsubsection{Análisis Factorial Exploratorio (AFE)}

El análisis factorial exploratorio implicó el desarrollo de diversos procedimientos. Primero, la prueba de esfericidad de Bartlett $\left({ }_{X}{ }^{2}(2628)=12287 p<.001\right)$ y la prueba de adecuación muestral de Kaiser-Meyer-Olkin (.909) respaldaron la idoneidad de los datos para la realización de un Análisis Factorial Exploratorio. Segundo, el análisis paralelo (Implementación óptima) permitió la identificación de 6 factores a extraer, los cuales explican un $48 \%$ de la varianza. El modelo presentó un ajuste adecuado, debido a que el valor de la raíz cuadrada media de los residuales $(\mathrm{RMSR})$ fue de $=.0434$, valor menor al esperado de .0538. Tercero, para la extracción de los factores se usó rotación oblicua Promin. En la matriz rotada de los ítems, se observó que algunos de ellos presentaban cargas factoriales inferiores al criterio mínimo de inclusión de .30, por lo que se decidió eliminar 7 ítems. La implementación nuevamente de los pasos indicados, esta vez con 63 ítems, permitió el perfeccionamiento del instrumento. Se volvieron a observar valores adecuados para las pruebas de Bartlett $\left({ }_{X}^{2}(1953)=10412.8 p<.001\right)$ y la prueba de adecuación muestral de Kaiser-Meyer-Olkin (.907). Se extrajeron 5 factores que explicaron un $48 \%$ de la varianza. El modelo presentó nuevamente un adecuado ajuste de la raíz cuadrada media de los residuales (RMSR) con un valor de .0455, inferior al valor esperado de .0538. La estructura resultante facilitó la definición de las dimensiones obtenidas.

El análisis conceptual de los ítems agrupados estadísticamente permitió la definición conceptual de los factores. Es importante indicar que el análisis factorial no permitió replicar los factores teóricos propuestos inicialmente, resultando una escala de 5 factores: i) Variedad de Uso; ii) Intensidad de Uso; iii) Multitarea; iv) Involucramiento; v) Dependencia. En el primer factor, Variedad de Uso, se agruparon todos los ítems que involucraban uso de las tecnologías en distintos tipos de actividades, como el ítem: "Durante el último mes he grabado y compartido en la red videos, fotos, audios de la familia, amigos, compañeros, etc.". En el segundo factor, Intensidad de Uso, se incluyeron ítems respecto a la dedicación diaria e intensa, por ejemplo: "Habitualmente utilizo Internet 4 o más horas diarias". En el tercer factor, Multitarea, se incluyeron ítems como "Prefiero hacer varias cosas a la vez, más que concentrar mis esfuerzos en una sola actividad”. En el cuarto factor, Involucramiento, se consideraron ítems que evidencian sentimientos de confianza cuando una persona piensa en sí misma en relación a las tecnologías, como "Me gusta aprender a usar nuevas tecnologías digitales" y sentimientos de compenetración con las tecnologías, como "Mi pertenencia a una comunidad digital es parte importante de mi identidad". Finalmente, en el quinto factor Dependencia se incluyeron ítems que aludían a acciones y /o expresiones que denotaban una gran vinculación a las tecnologías, por ejemplo: "Siento que no puedo estar ningún día sin Internet”. Esta solución dejó afuera algunos factores inicialmente considerados.

Los factores Amplitud, Focalización Preferencial, Compenetración y Compromiso Emocional fueron excluidos de la versión final de la escala. Los ítems que fueron construidos para el concepto de Amplitud se distribuyeron en el factor Intensidad de Uso e Involucramiento, en tanto los ítems de Focalización Preferencial se posicionaron 
estadísticamente en el factor Dependencia. De igual manera, los ítems de los factores Compenetración y Compromiso Emocional se fusionaron en el nuevo factor denominado Involucramiento. Esta redistribución fue analizada teóricamente y aceptada como coherente por el equipo de investigación. La Tabla 3 presenta información respecto a la confiabilidad de los factores (alfa de Cronbach en primera instancia), números de ítems y rangos de carga factorial de cada factor.

Tabla 3. Factores obtenidos en AFE Escala de Inmersión Digital n=347

\begin{tabular}{|l|c|c|c|}
\hline Factor & Alfa $^{*}$ & $\mathrm{~N}^{\mathrm{o}}$ Ítems & Rangos de carga factorial \\
\hline Variedad de Uso & .831 & 10 & $.400-.773$ \\
\hline Intensidad de Uso & .860 & 13 & $.374-.744$ \\
\hline Multitarea & .836 & 8 & $.435-.816$ \\
\hline Involucramiento & .922 & 18 & $.508-.784$ \\
\hline Dependencia & .909 & 13 & $.569-756$ \\
\hline
\end{tabular}

* Se reporta el alfa de Cronbach estandarizado reportado por el programa FACTOR.

\subsubsection{Análisis Factorial Confirmatorio (AFC)}

A través de diversas interacciones, utilizando análisis factorial confirmatorio, se obtuvo una solución final de la escala. Para la obtención de esta solución fue necesario eliminar algunos ítems, lo que permitió obtener mejores indicadores de bondad de ajuste. Además, se optó por descartar otros ítems que permitieran balancear el número de ítems por factor facilitando implícitamente la aplicación del cuestionario en términos de menor tiempo empleado para dicha tarea. Como criterios de eliminación se consideró que los ítems tuviesen una menor carga factorial y/o tuviesen una alta covarianza de su error con el error de otro ítem (aspecto que evidenciaba que ambos ítems medían aspectos similares). La escala total quedó compuesta por 30 ítems, cinco de ellos pertenecientes a la dimensión Variedad de Uso, siete a la dimensión Intensidad de Uso, seis a la dimensión Multitarea, siete a la dimensión Involucramiento y cinco a la dimensión Dependencia. Es importante indicar que la estructura teóricamente identificada en el análisis factorial exploratorio fue confirmada en este análisis confirmatorio.

La Tabla 4 presenta los factores, ítems y las cargas factoriales de los ítems definitivos vinculados a los 5 factores: 
Tabla 4. Factores e ítems de la Escala de Inmersión Digital para Adolescentes (EIDA)

\begin{tabular}{|c|c|c|}
\hline Factor & $\lambda$ & Ítems \\
\hline \multirow{5}{*}{ Variedad de Uso } & .748 & $\begin{array}{l}\text { 01. Durante el último mes he posteado notas o imágenes } \\
\text { de interés (por ejemplo, en Facebook o en un blog). }\end{array}$ \\
\hline & .693 & $\begin{array}{l}\text { 06. Durante el último mes he grabado y compartido en la } \\
\text { red videos, fotos, audios de la familia, amigos, } \\
\text { compañeros, etc. }\end{array}$ \\
\hline & .654 & 11. Durante el último mes he actualizado mi perfil. \\
\hline & .589 & $\begin{array}{l}\text { 16. Durante el último mes he editado fotos o videos } \\
\text { usando tecnologías. }\end{array}$ \\
\hline & .497 & 21. Todos los días "chateo". \\
\hline \multirow{7}{*}{ Intensidad de Uso } & .682 & 02. Habitualmente utilizo Internet 4 o más horas diarias. \\
\hline & .675 & $\begin{array}{l}\text { 07. Todos los días escucho música o veo películas en } \\
\text { línea. }\end{array}$ \\
\hline & .627 & 12. Todos los días utilizo Internet para buscar información. \\
\hline & .621 & $\begin{array}{l}\text { 17. Me mantengo en contacto con mis amigos todos los } \\
\text { días a través de Internet. }\end{array}$ \\
\hline & .593 & $\begin{array}{l}\text { 22. Todos los días me conecto a Internet desde más de un } \\
\text { dispositivo como celular, computador, Tablet, entre otros. }\end{array}$ \\
\hline & .537 & $\begin{array}{l}\text { 26. Uso diariamente una variada gama de dispositivos } \\
\text { como celular, computador, Tablet, Smart TV entre otros. }\end{array}$ \\
\hline & .516 & 29. He usado Internet al menos los últimos 4 años. \\
\hline \multirow{6}{*}{ Multitarea } & .845 & $\begin{array}{l}\text { 03. Prefiero hacer varias cosas a la vez, más que } \\
\text { concentrar mis esfuerzos en una sola actividad. }\end{array}$ \\
\hline & .735 & $\begin{array}{l}\text { 08. Cuando tengo que hacer varias tareas, prefiero } \\
\text { alternarlas en vez de hacer una a la vez. }\end{array}$ \\
\hline & .703 & $\begin{array}{l}\text { 13. Prefiero trabajar en varias cosas a la vez, en lugar de } \\
\text { terminar una y luego pasar a la otra. }\end{array}$ \\
\hline & .667 & $\begin{array}{l}\text { 18. Me entretiene más si puedo ir cambiando entre varias } \\
\text { tareas diferentes. }\end{array}$ \\
\hline & .426 & $\begin{array}{l}\text { 23. Pierdo interés en lo que estoy haciendo si tengo que } \\
\text { concentrarme en la misma tarea por mucho tiempo, sin } \\
\text { poder pensar o hacer otra cosa. }\end{array}$ \\
\hline & .423 & $\begin{array}{l}\text { 27. Cuando tengo que completar una tarea, me gusta } \\
\text { intercalar algunas pausas para hacer otras cosas. }\end{array}$ \\
\hline
\end{tabular}




\begin{tabular}{|c|c|c|}
\hline \multirow{7}{*}{ Involucramiento } & .740 & $\begin{array}{l}\text { 04. Me gusta explorar innovaciones tecnológicas que me } \\
\text { faciliten la vida. }\end{array}$ \\
\hline & .685 & $\begin{array}{l}\text { 09. Cuando sale una nueva versión de una aplicación, la } \\
\text { busco y actualizo todos mis equipos. }\end{array}$ \\
\hline & 665 & $\begin{array}{l}\text { 14. Me siento tranquilo/a si me piden hacer algo nuevo } \\
\text { usando tecnologías. }\end{array}$ \\
\hline & 661 & $\begin{array}{l}\text { 19. Me gusta mucho probar nuevas aplicaciones y } \\
\text { funcionalidades digitales. }\end{array}$ \\
\hline & 647 & $\begin{array}{l}\text { 24. Me gusta hacer muchas cosas usando computadores o } \\
\text { celulares. }\end{array}$ \\
\hline & 641 & $\begin{array}{l}\text { 28. Entre mis amigos y compañeros, siempre soy el } \\
\text { primero en conocer las novedades en tecnología. }\end{array}$ \\
\hline & 627 & 30. Me siento parte de una nueva cultura digital. \\
\hline \multirow{5}{*}{ Dependencia } & .722 & $\begin{array}{l}\text { 05. Cuanto estoy usando Internet me concentro totalmente } \\
\text { en lo que estoy haciendo. }\end{array}$ \\
\hline & 693 & $\begin{array}{l}\text { 10. No me gusta que me interrumpan cuando estoy } \\
\text { usando Internet. }\end{array}$ \\
\hline & .650 & 15. Casi nada me distrae cuando estoy usando Internet. \\
\hline & 630 & $\begin{array}{l}\text { 20. El uso de Internet ocupa una porción importante de } \\
\text { mis actividades diarias. }\end{array}$ \\
\hline & .423 & 25. Puedo olvidar mi billetera, pero nunca mi celular. \\
\hline
\end{tabular}

Fuente. Elaboración propia.

La Figura 1 muestra la solución factorial obtenida en la escala de Inmersión Digital. Como se puede observar, posterior a la ejecución del test de Lagrange, se incorporó al modelo una covarianza entre dos ítems (21. Todos los días "chateo" y 17. Me mantengo en contacto con mis amigos todos los días a través de Internet). Aunque dichos ítems corresponden a factores distintos, claramente están relacionados, por lo que se decidió conservar dicha covarianza. 
Figura 1. Solución factorial Escala de Inmersión Digital para Adolescentes (EIDA).

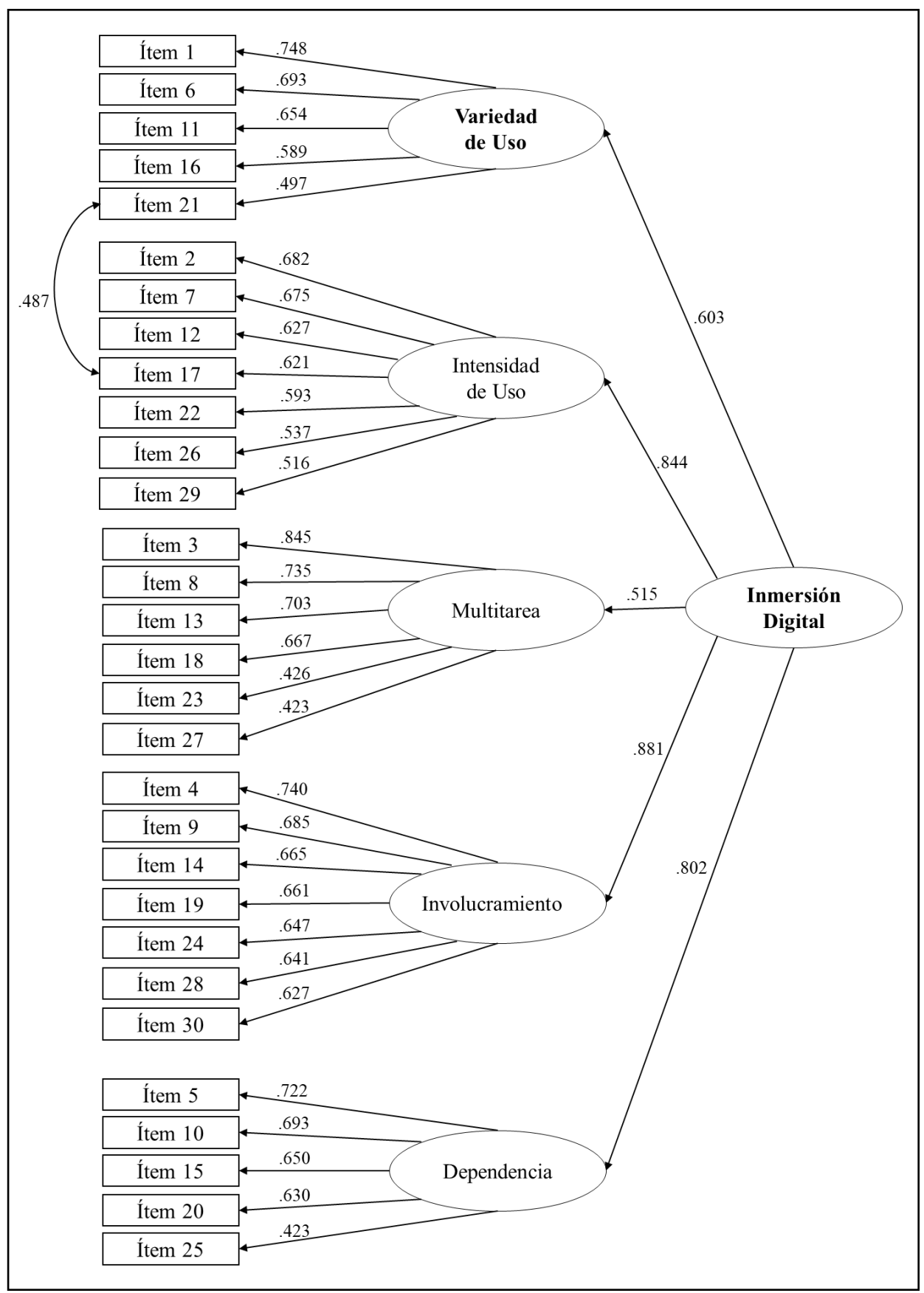

Fuente: Elaboración propia. 
Por otra parte, los indicadores de bondad de ajuste del modelo global presentan resultados adecuados $($ SRMR $=.065$, TLI_SB $=.910$, CFI $=.918$, RMSEA $=.043)$, explicando la solución obtenida un $90 \%$ de la varianza $\left(\mathrm{R}^{2}=.896\right)$. Coincidente con esto, los indicadores de cada factor presentaron valores aceptables, tal como se puede observar en la Tabla 5.

Tabla 5. Niveles de bondad de ajuste factores Escala de Inmersión Digital $n=347$

\begin{tabular}{|l|c|c|c|c|c|}
\hline Factor & $\mathrm{X}^{2} \mathrm{sb} \_\mathrm{ms}$ & RMSEA_SB & CFI_SB & TLI_SB & SRMR \\
\hline Variedad de Uso & $8.103(5)$ & .042 & .992 & .984 & .023 \\
\hline Intensidad de Uso & $11.579(14)$ & .000 & 1.000 & 1.000 & .020 \\
\hline Multitarea & $29.701(9)$ & .006 & .976 & .960 & .035 \\
\hline Involucramiento & $9.868(14)$ & .000 & 1.000 & 1.000 & .019 \\
\hline Dependencia & $11.194(5)$ & .060 & .982 & .964 & .026 \\
\hline
\end{tabular}

Fuente. Elaboración propia.

\subsection{CONFIABILIDAD: CONSISTENCIA INTERNA}

La confiabilidad total de la escala presenta un valor alfa ordinal de .951, lo que se considera un nivel adecuado para una escala (Llarena, 2008). Asimismo, cada factor presentó valores alfa ordinal adecuados, tal como se puede observar en la Tabla 6:

Tabla 6. Niveles de bondad de ajuste factores Escala de Inmersión Digital para Adolescentes (EIDA)

\begin{tabular}{|l|c|c|}
\hline \multicolumn{1}{|c|}{ Factor } & Alfa ordinal & $\mathrm{N}^{\circ}$ ítems \\
\hline Variedad de Uso & .816 & 5 \\
\hline Intensidad de Uso & .848 & 7 \\
\hline Multitarea & .825 & 6 \\
\hline Involucramiento & .866 & 7 \\
\hline Dependencia & .788 & 5 \\
\hline
\end{tabular}

\section{DISCUSIÓN Y CONCLUSIONES}

Este estudio tuvo como objetivo construir y analizar psicométricamente una escala para medir inmersión digital en adolescentes. En cuanto a validez de contenido, hubo un alto nivel de consenso entre los jueces respecto a la capacidad de los ítems de medir los factores 
incluidos en la escala. Esta primera acción, permitió eliminar algunos ítems, agregar otros y corregir la redacción. Siendo un paso importante para la elaboración de la escala, en tanto permitió conocer la opinión de expertos que trabajan en el uso que los jóvenes desarrollan con las tecnologías.

En relación a las evidencias de validez de la estructura interna, se realizaron AFE y AFC. El AFE permitió observar una estructura factorial de 5 factores. Dicha estructura no se ajustó a lo que teóricamente se había planteado en la construcción de esta escala, pero sí permitió reformular los factores planteados, ajustando la teoría con lo empíricamente observado. El AFC permitió confirmar la estructura obtenida en el AFE, dando cuenta que la escala de inmersión digital está compuesta por 5 factores: 1) Variedad de Uso de las Tecnologías; 2) Intensidad de Uso de las Tecnologías; 3) Multitarea; 4) Involucramiento; y 5) Dependencia. Estos resultados dan sustento para afirmar que, es posible medir el constructo inmersión digital en adolescentes. Pese a que el instrumento desarrollado presenta diferencias en los factores que lo componen con respecto a cómo originalmente fueron planteados.

Medir inmersión digital no es una tarea sencilla debido a que es un constructo de reciente data. En coherencia con lo planteado en los antecedentes teóricos, el concepto de inmersión digital incluye aspectos que Rosenfeld (2015), Owston (2009) y Jenkins (2006) no habían considerado. Pues dichos autores presentaban una definición de inmersión digital focalizada en la intensidad y variedad del uso de las tecnologías digitales, los dos primeros factores esta escala. De igual manera esta investigación demostró empíricamente que la inmersión digital incluye otros factores como multitarea. Dicho factor, fue mencionado inicialmente por Prensky (2001) en relación a los nativos digitales, indicando que los jóvenes que crecen en ambientes multimediales e hiperconectados se ven estimulados en su plasticidad cerebral, desarrollando una capacidad para involucrarse en múltiples tareas. Por otra parte, en esta escala se incluyeron también los factores involucramiento y dependencia, constructos más vinculados al concepto de inmersión asociado a los videojuegos, mundos virtuales y realidad virtual. El factor involucramiento puede relacionarse con lo planteado por Ryan (2008), quien considera la inmersión como un estado intenso de absorción en la tarea que se realiza. Mientras que, el factor de Dependencia, se asemeja a la conceptualización de inmersión planteada por Ermi y Mäyrä (2005), quienes definen dependencia como la sensación de estar mentalmente absorto.

En relación a la dimensión original de identidad tecnológica, se destaca que fue planteada teóricamente por Carter y Grover (2015), incluyendo las sub dimensiones Compenetración, Compromiso Emocional y Dependencia. Sin embargo, en este estudio se fusionaron los ítems de dichas sub dimensiones. Al respecto, sería interesante analizar en futuros estudios si la identidad tecnológica como constructo tiene un correlato empírico con las tres sub dimensiones planteadas por dichos autores o si, como sucedió en este estudio, se remite a dos factores.

Por otra parte, con respecto a la confiabilidad de la escala el instrumento presenta buenos niveles de consistencia interna. Esto puede ser observado en los valores para la escala completa $(\alpha=.951)$, así como para cada uno de los factores evaluados (valores alfa entre $\alpha=.788$ y .866). Lo que da cuenta de que esta escala es confiable, es decir, al repetir las mediciones en muestras y circunstancias similares, se producirán resultados iguales o parecidos (Abad et al., 2011).

En cuanto a las limitaciones del estudio, es necesario considerar que todos los participantes eran adolescentes chilenos de la región de La Araucanía y aunque se trató de 
incluir diversas características sociodemográficas, como sexo, curso, nivel socioeconómico y etnia, siguen perteneciendo a una misma región de un país. Es por dicha razón que se considera necesario realizar futuros estudios donde se confirme tanto la estructura factorial como la consistencia interna con otras muestras. Además, se espera que en futuros estudios esta escala sea aplicada a otras poblaciones de adolescentes, para así poder realizar comparaciones y conocer si efectivamente se cumplen en otros contextos socioculturales los resultados obtenidos con los adolescentes chilenos. Otra limitación está relacionada con la obtención de mayores evidencias de validez de esta escala. Es por eso que se espera -a futuro- poder desarrollar estudios con alguna escala similar para obtener evidencias de validez convergente y con medidas de constructos diferentes para obtener evidencias de validez discriminante (Elosua, 2003). De esta forma se podría obtener evidencias de validez externa, donde los datos disponibles en conjunto con la teoría apoyen la interpretación de las puntuaciones de la escala para poder evaluar la inmersión digital de los adolescentes.

Por otra parte, un aspecto relevante a analizar a futuro es la relación entre distintas medidas de aprendizaje autodirigido (Cerda et al., 2015) y la inmersión digital. Gran parte de la inmersión digital se desarrolla de manera autónoma en los usuarios, sea esto con propósitos académicos, recreativos o sociales que van generando distintos niveles de inmersión según el propósito de uso a desarrollar, por lo que sería interesante profundizar en futuros estudios dichas temáticas (Cerda et al., 2018).

Complementariamente, se podría profundizar en los efectos que tendrían los distintos niveles de inmersión digital. Esta tarea se podría llevar a cabo estudiando algunas características de los adolescentes que presentan un bajo, medio o alto nivel de inmersión en procesos cognitivos como la atención, funciones ejecutivas u otros procesos que se consideren relevantes, como el estudio del uso de entornos virtuales de aprendizaje (SilvaQuiroz et al., 2016) o su relación con el bienestar psicológico (Capilla y Cubo, 2017). Además, se podría focalizar en el nivel de inmersión digital y sus efectos en la salud de niños y jóvenes, de este modo se podrían desarrollar estrategias e intervenciones en relación con el uso adecuado y responsable de las tecnologías digitales para la promoción de la salud y la prevención de conductas de riesgo (Muñoz-Miralles et al., 2014).

Actualmente se observa que, a pesar de la amplitud y densidad de acceso a las tecnologías digitales por parte de los adolescentes, los parámetros de uso no son homogéneos (Calvani et al., 2012). Por tanto, puede inferirse que podrían existir diversos niveles de inmersión digital de los adolescentes. De acuerdo a van Deursen y Helsper (2015), las personas de grupos sociales más aventajados obtienen mayores beneficios de usar Internet. En los resultados de la prueba ICILS (International Computer and Information Literacy Study) se observó que cuando el ingreso económico de las familias era alto, los estudiantes tenían mejores desempeños (Fraillon et al., 2014). Complementariamente, Zillien y Hargittai (2009) reportan que cuando los usuarios tenían un ingreso económico más alto realizaban actividades en línea de mayor capital cultural que las personas de ingresos bajos. Observándose también un menor uso de las tecnologías en personas con menor ingreso económico (Hargittai, 2010) y que los jóvenes con alto capital cultural son quienes tienen mayor capacidad de adquirir conocimientos y desarrollar sus hobbies usando tecnologías (Matamala, 2016).

Para concluir, se pone a disposición de la comunidad científica una escala con una adecuada consistencia interna y estructura factorial, idónea para evaluar el nivel de inmersión digital de los adolescentes. Se espera que con esta escala se pueda contribuir a 
evaluar los profundos cambios culturales en la vida de adolescentes y jóvenes en la sociedad actual, profundamente marcados por el uso de las tecnologías digitales. Estos cambios se pueden entender como el surgimiento de una nueva cultura digital y lo que se construyó en este estudio es una medida del grado de inmersión de estos jóvenes en su cultura digital.

\section{REFERENCIAS BIBLIOGRÁFICAS}

Abad, F., Olea, J., Ponsoda, V. \& García, C. (2011). Medición en ciencias sociales y de la salud. Síntesis.

Adams, E. \& Rollings, A. (2006). Fundamentals of game design. Prentice Hall.

Boyd, D. (2014). It's complicated: The social lives of networked teens. Yale University Press.

Bruni, L. E. (2011). Cognitive sustainability in the age of digital culture. TripleC: Communication, Capitalism \& Critique. Open Access Journal for a Global Sustainable Information Society, 9(2), 473-482. https://doi.org/10.31269/vol9iss2pp473-482

Calvani, A., Fini, A., Ranieri, M. \& Picci, P. (2012). Are young generations in secondary school digitally competent? A study on Italian teenagers. Computers \& Education, 58(2), 797-807. https://doi.org/10.1016/j.compedu.2011.10.004

Capilla, E. \& Cubo, S. (2017). Phubbing. Conectados a la red y desconectados de la realidad. Un análisis en relación al bienestar psicológico. Pixel-Bit. Revista de Medios y Educación (50), 173185. https://doi.org/10.12795/pixelbit.2017.i50.12

Carter, M. \& Grover, V. (2015). Me, my self and I(T): Conceptualizing information technology identity and its implications. MIS Quarterly, 39(4), 931-957. https://doi.org/10.25300/ MISQ/2015/39.4.9

Cerda, C., López, Ó., Osses, S. \& Saiz, J. L. (2015). Análisis psicométrico de la escala de aprendizaje autodirigido basada en la teoría de aprendizaje autodirigido de Garrison. Revista Iberoamericana de Diagnóstico y Evaluación-e Avaliação Psicológica, 1(39), 46-56. https://bit.ly/2NpQuq2

Cerda, C., Saiz, J. L., Villegas, L. \& León, M. (2018). Acceso, tiempo y propósito de uso de tecnologías digitales en estudiantes de pedagogía chilenos. Estudios Pedagógicos, 44(3), 7-22. https://doi.org/10.4067/S0718-07052018000300007

Courage, M. L., Bakhtiar, A., Fitzpatrick, C., Kenny, S. \& Brandeau, K. (2015). Growing up multitasking: The costs and benefits for cognitive development. Developmental Review, 35, 5-41. https://doi.org/10.1016/j.dr.2014.12.002

Domínguez, S. (2012). Propuesta para el cálculo del alfa ordinal y theta de Armor. Revista de Investigación en Psicología, 15(1), 213-217. https://doi.org/10.15381/rinvp.v15i1.3684

Dominic, A. (2005). Dark waters: Spotlight on immersion. Trabajo presentado en la conferencia Dans GAMEON-NA North America 2005 International Conference, Montreal, Canadá. https:// papyrus.bib.umontreal.ca/xmlui/handle/1866/13052

Elosua, P. (2003). Sobre la validez de los tests. Psicothema, 15(2), 315-321. http://www.psicothema. es/pdf/1063.pdf

Ermi, L. \& Mäyrä, F. (2005). Fundamental components of the gameplay experience: Analysing immersion. En S. Castell \& J. Jenson (Eds.), Worlds in play: International perspectives on digital games research (pp. 37-53). Peter Lang Publishing.

Flora, D. B. \& Curran, P. J. (2004). An empirical evaluation of alternative methods of estimation for confirmatory factor analysis with ordinal data. Psychological Methods, 9(4), 466-491. https:// doi.org/10.1037/1082-989x.9.4.466

Fraillon, J., Ainley, J., Schulz, W., Friedman, T. \& Gebhardt, E. (2014). Preparing for life in a digital age: The IEA International Computer and Information Literacy Study international report. Springer. 
Georgiou, Y. \& Kyza, E. A. (2017). The development and validation of the ARI questionnaire: An instrument for measuring immersion in location-based augmented reality settings. International Journal of Human-Computer Studies, 98, 24-37. https://doi.org/10.1016/j.ijhcs.2016.09.014

Gere, C. (2008). Digital culture. Reaktion Books.

Giedd, J. N. (2012). The digital revolution and adolescent brain evolution. Journal of Adolescent Health, 51(2), 101-105. https://doi.org/10.1016/j.jadohealth.2012.06.002

Hargittai, E. (2010). Digital na(t)ives? Variation in Internet skills and uses among members of the "Net Generation". Sociological Inquiry, 80(1), 92-113. https://doi.org/10.1111/j.1475682x.2009.00317.x

Ito, M., Antin, J., Finn, M., Law, A., Manion, A., Mitnick, S., . . Horst, H. A. (2009). Hanging out, messing around, and geeking out: Kids living and learning with new media. The MIT Press.

Jenkins, H. (2006). Convergence culture: Where old and new media collide. New York University Press.

Llarena, M. (2008). Metodología para la evaluación de la calidad de estrategias didácticas de cursos a distancia (MACCAD). Formación Universitaria, 1(2), 37-46. https://doi.org/10.4067/S071850062008000200006

Lorenzo-Seva, U. (1999). Promin: A method for oblique factor rotation. Multivariate Behavioral Research, 34(3), 347-365. https://doi.org/10.1207/S15327906MBR3403_3

Lorenzo-Seva, U. \& Ferrando, P. (2006). FACTOR: A computer program to fit the exploratory factor analysis model. Behavior Research Methods, 38(1), 88-91. https://doi.org/10.3758/bf03192753

Matamala, C. (2016). Uso de las TIC en el hogar: Entre el entretenimiento y el aprendizaje informal. Estudios Pedagógicos, 42(3), 293-311. https://doi.org/10.4067/S0718-07052016000400016

McGraw, K. O. \& Wong, S. P. (1996). Forming inferences about some intraclass correlation coefficients. Psychological Methods, 1(1), 30-46. https://doi.org/10.1037/1082-989X.1.1.30

Muñiz, J. \& Fonseca-Pedrero, E. (2008). Construcción de instrumentos de medida para la evaluación universitaria. Revista de Investigación en Educación, 5, 13-25. https://bit.ly/2Q3sWbZ

Muñoz-Miralles, R., Ortega-González, R., Batalla-Martínez, C., López-Morón, M. R., Manresa, J. M. \& Torán-Monserrat, P. (2014). Acceso y uso de nuevas tecnologías entre los jóvenes de educación secundaria, implicaciones en salud. Estudio JOITIC. Atención Primaria, 46(2), $77-$ 88. https://doi.org/10.1016/j.aprim.2013.06.001

Murray, J. (1997). Hamlet on the holodeck: The future of narrative in cyberspace. MIT Free Press.

Nilsson, N. C., Nordahl, R. \& Serafin, S. (2016). Immersion revisited: A review of existing definitions of immersion and their relation to different theories of presence. Human Technology, 12(2), 108134. https://doi.org/10.17011/ht/urn.201611174652

Owston, R. D. (2009). Comments on Greenhow, Robelia, and Hughes: Digital immersion, teacher learning, and games. Educational Researcher, 38(4), 270-273. https://doi. org/10.3102/0013189x09336673

Poposki, E. M. \& Oswald, F. L. (2010). The multitasking preference inventory: Toward an improved measure of individual differences in polychronicity. Human Performance, 23(3), 247-264. https://doi.org/10.1080/08959285.2010.487843

Prensky, M. (2001). Digital natives, digital immigrants. On the Horizon, 9(5), 1-6. https://doi. org/10.1108/10748120110424816

Rosenfeld, K. N. (2015). Terms of the digital age: Realities and cultural paradigms. En M. N. Yildiz \& J. Keengwe (Eds.). Handbook of Research on Media Literacy in the Digital Age (p.115-136). IGI Global

Rosner, B. (2011). Fundamentals of biostatistics (6a ed.). Brooks/Cole.

Ryan, M. L. (2008). Interactive narrative, plot types, and interpersonal relations. En U. Spierling \&N. Szilas (Eds) Interactive Storytelling. ICIDS 2008. Lecture Notes in Computer Science, vol 5334. (pp. 6-13). Springer.

Shapiro, A. \& Berge, J. F. (2002). Statistical inference of minimum rank factor analysis. Psychometrika, 67(1), 79-94. https://doi.org/10.1007/bf02294710 
Silva-Quiroz, J., Serrano, E. F. \& Cavieres, A. A. (2016). Modelo interactivo en red para el aprendizaje: Hacia un proceso de aprendizaje online centrado en el estudiante. Pixel-Bit. Revista de Medios y Educación, (49), 225-238. https://doi.org/10.12795/pixelbit.2016.i49.015

Slater, M. (2003). A note on presence terminology. Presence Connect, 3(3), 1-5. https://bit. $1 \mathrm{y} / 32 \mathrm{FOzC} 8$

Timmerman, M. E. \& Lorenzo-Seva, U. (2011). Dimensionality assessment of ordered polytomous items with parallel analysis. Psychological Methods, 16(2), 209-220. https://doi.org/10.1037/ a0023353

van Deursen, A. J. \& Helsper, E. (2015). A nuanced understanding of Internet use and nonuse among the elderly. European Journal of Communication, 30(2), 171-187. https://doi. org/10.1177/0267323115578059

van Deursen, A. J., Helsper, E. \& Eynon, R. (2014). Measuring digital skills. From digital skills to tangible outcomes. Project report. https://bit.ly/2Cg0yeP

Zillien, N. \& Hargittai, E. (2009). Digital distinction: Status-specific types of Internet usage. Social Science Quarterly, 90(2), 274-291. https://doi.org/10.1111/j.1540-6237.2009.00617.x

Zumbo, B. D., Gadermann, A. M. \& Zeisser, C. (2007). Ordinal versions of coefficients alpha and theta for Likert rating scales. Journal of Modern Applied Statistical Methods, 6(1), 21-29. https://doi.org/10.22237/jmasm/1177992180 


\title{
Escala de Inmersión Digital para Adolescentes (EIDA)
}

\author{
(León, M., Cerda, C., Rehbein, L. \& Saiz, J.)
}

Indica qué tan de acuerdo o en desacuerdo estás con las siguientes afirmaciones. Marca con una X.

\begin{tabular}{|c|c|c|c|c|}
\hline $\begin{array}{c}\mathbf{1} \\
\text { Muy en } \\
\text { desacuerdo }\end{array}$ & $\begin{array}{c}\mathbf{2} \\
\text { En desacuerdo }\end{array}$ & $\begin{array}{c}\text { Ni de acuerdo ni } \\
\text { en desacuerdo }\end{array}$ & $\begin{array}{c}\mathbf{4} \\
\text { De acuerdo }\end{array}$ & $\begin{array}{c}\mathbf{5} \\
\text { Muy de acuerdo }\end{array}$ \\
\hline
\end{tabular}

\begin{tabular}{|c|c|c|c|c|c|}
\hline $\begin{array}{l}\text { 01. Durante el último mes he posteado notas o imágenes de interés (por } \\
\text { ejemplo, en Facebook o en un blog). }\end{array}$ & 1 & 2 & 3 & 4 & 5 \\
\hline 02. Habitualmente utilizo Internet 4 o más horas diarias. & 1 & 2 & 3 & 4 & 5 \\
\hline $\begin{array}{l}\text { 03. Prefiero hacer varias cosas a la vez, más que concentrar mis } \\
\text { esfuerzos en una sola actividad. }\end{array}$ & 1 & 2 & 3 & 4 & 5 \\
\hline 04. Me gusta explorar innovaciones tecnológicas que me faciliten la vida. & 1 & 2 & 3 & 4 & 5 \\
\hline $\begin{array}{l}\text { 05. Cuanto estoy usando Internet me concentro totalmente en lo que } \\
\text { estoy haciendo. }\end{array}$ & 1 & 2 & 3 & 4 & 5 \\
\hline $\begin{array}{l}\text { 06. Durante el último mes he grabado y compartido en la red videos, } \\
\text { fotos, audios de la familia, amigos, compañeros, etc. }\end{array}$ & 1 & 2 & 3 & 4 & 5 \\
\hline 07. Todos los días escucho música o veo películas en línea. & 1 & 2 & 3 & 4 & 5 \\
\hline $\begin{array}{l}\text { 08. Cuando tengo que hacer varias tareas, prefiero alternarlas en vez de } \\
\text { hacer una a la vez. }\end{array}$ & 1 & 2 & 3 & 4 & 5 \\
\hline $\begin{array}{l}\text { 09. Cuando sale una nueva versión de una aplicación, la busco y } \\
\text { actualizo todos mis equipos. }\end{array}$ & 1 & 2 & 3 & 4 & 5 \\
\hline 10. No me gusta que me interrumpan cuando estoy usando Internet. & 1 & 2 & 3 & 4 & 5 \\
\hline 11. Durante el último mes he actualizado mi perfil. & 1 & 2 & 3 & 4 & 5 \\
\hline 12. Todos los días utilizo Internet para buscar información. & 1 & 2 & 3 & 4 & 5 \\
\hline $\begin{array}{l}\text { 13. Prefiero trabajar en varias cosas a la vez, en lugar de terminar una } \\
\text { y luego pasar a la otra. }\end{array}$ & 1 & 2 & 3 & 4 & 5 \\
\hline $\begin{array}{l}\text { 14. Me siento tranquilo/a si me piden hacer algo nuevo usando } \\
\text { tecnologías. }\end{array}$ & 1 & 2 & 3 & 4 & 5 \\
\hline 15. Casi nada me distrae cuando estoy usando Internet. & 1 & 2 & 3 & 4 & 5 \\
\hline 16. Durante el último mes he editado fotos o videos usando tecnologías. & 1 & 2 & 3 & 4 & 5 \\
\hline $\begin{array}{l}\text { 17. Me mantengo en contacto con mis amigos todos los días a través de } \\
\text { Internet. }\end{array}$ & 1 & 2 & 3 & 4 & 5 \\
\hline $\begin{array}{l}\text { 18. Me entretiene más si puedo ir cambiando entre varias tareas } \\
\text { diferentes. }\end{array}$ & 1 & 2 & 3 & 4 & 5 \\
\hline $\begin{array}{l}\text { 19. Me gusta mucho probar nuevas aplicaciones y funcionalidades } \\
\text { digitales. }\end{array}$ & 1 & 2 & 3 & 4 & 5 \\
\hline $\begin{array}{l}\text { 20. El uso de Internet ocupa una porción importante de mis actividades } \\
\text { diarias. }\end{array}$ & 1 & 2 & 3 & 4 & 5 \\
\hline
\end{tabular}




\begin{tabular}{|l|c|c|c|c|c|}
\hline 21. Todos los días "chateo". & 1 & 2 & 3 & 4 & 5 \\
\hline $\begin{array}{l}\text { 22. Todos los días me conecto a Internet desde más de un dispositivo } \\
\text { como celular, computador, Tablet, entre otros. }\end{array}$ & 1 & 2 & 3 & 4 & 5 \\
\hline $\begin{array}{l}\text { 23. Pierdo interés en lo que estoy haciendo si tengo que concentrarme } \\
\text { en la misma tarea por mucho tiempo, sin poder pensar o hacer otra } \\
\text { cosa. }\end{array}$ & 1 & 2 & 3 & 4 & 5 \\
\hline 24. Me gusta hacer muchas cosas usando computadores o celulares. & 1 & 2 & 3 & 4 & 5 \\
\hline $\begin{array}{l}\text { 25. Puedo olvidar mi billetera, pero nunca mi celular. } \\
\text { 26. Uso diariamente una variada gama de dispositivos como celular, } \\
\text { computador, Tablet, Smart TV entre otros. }\end{array}$ & 1 & 2 & 3 & 4 & 5 \\
\hline $\begin{array}{l}\text { 27. Cuando tengo que completar una tarea, me gusta intercalar algunas } \\
\text { pausas para hacer otras cosas. }\end{array}$ & 1 & 2 & 3 & 4 & 5 \\
\hline $\begin{array}{l}\text { 28. Entre mis amigos y compañeros, siempre soy el primero en conocer } \\
\text { las novedades en tecnología. }\end{array}$ & 1 & 2 & 3 & 4 & 5 \\
\hline \begin{tabular}{l} 
29. He usado Internet al menos los últimos 4 años. \\
\hline 30. Me siento parte de una nueva cultura digital.
\end{tabular} & 1 & 2 & 3 & 4 & 5 \\
\hline
\end{tabular}


